\title{
Heavy quark mass effects in associated production
}

\section{Davide Napoletano*}

IPhT, CEA Saclay, CNRS UMR 3681, F-91191, Gif-Sur-Yvette, France

E-mail: davide.napoletano@ipht.fr

\begin{abstract}
Processes involving heavy quarks in associated production, are usually described in two factorisation schemes: one in which the heavy quark is treated as an infinitely massive object, decoupled from QCD evolution, and one in which it is treated on the same footing of a light quark. These two approaches only differ by the inclusion of mass suppressed terms or in the resummation of a certain class of logarithms. In view of recent results, in this talk, we present recent developments that extend a phenomenological scheme, that tries to incorporate the best of both worlds, to NLO in Monte Carlo event generation.
\end{abstract}

XXVI International Workshop on Deep-Inelastic Scattering and Related Subjects (DIS2018) 16-20 April 2018

Kobe, Japan

${ }^{*}$ Speaker. 
The production of heavy quarks in association with a vector boson, or a Higgs boson, has re-attracted interest from the theory community in recent years $[1,2,3,4,5,6,7,8,9,10,11]$ for essentially two reasons. Firstly, they constitute an irreducible background for many Higgs processes, both in the Standard Model and beyond, which requires good theoretical control for calculations of such processes. The second, and more theoretical reason, comes from the fact that these processes involve at least two, well separated, scales. As it is well known, this manifests itself, in QCD processes, through logarithms of ratios of these scales, which one may or may not want to resum.

When, in particular, $b$ quarks are excited from the proton, and thus appear in the initial state, two factorisation schemes are usually employed, the 4-flavour and the 5-flavour schemes. In the 4-flavour scheme, the $b$ quark is considered as an infinitely massive object decoupled from QCD evolution. In this approach, then, it can only be produced in the final state, through a $g \rightarrow b \bar{b}$ splitting if the gluon has enough energy. Fixed-order logarithms of any energy-like invariant over $m_{b}$ can appear. In the 5-flavour scheme $b$ quarks have zero mass, and thus participate in the QCD evolution, like all other light quarks. $b$-mass effects, appear only as threshold effects, and logarithms of $\mu_{F} / m_{b}$ are resummed to all order.
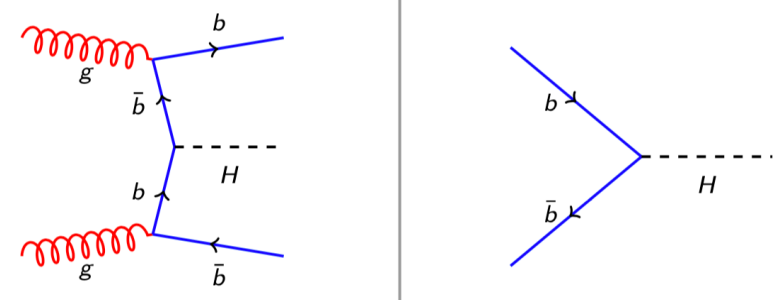

Figure 1: Sketched comparison of the 4F and the 5F scheme.

In inclusive enough observables, and easy enough processes, like in the calculation of the total inclusive cross section in $b \bar{b} \rightarrow H / Z$, setting $\mu_{F}$ in the 5 flavour scheme to the value of the hard scale in the four flavour scheme, one can easily see the correspondence between the two schemes (Fig. 1) and construct a matching scheme to combine the two and obtain a result which includes both fixed-order mass effects, and the resummation of large $\mu_{F} / m_{b}$ logarithms $[3,4,6,8,11]$.

These matched results can give insights on what are the main contributions that make the two factorisation schemes different: mass or resummation effects. Looking, for example, at the top row of Fig. 2, one can see that the matched result, both with leading or next-to-leading fixed order 

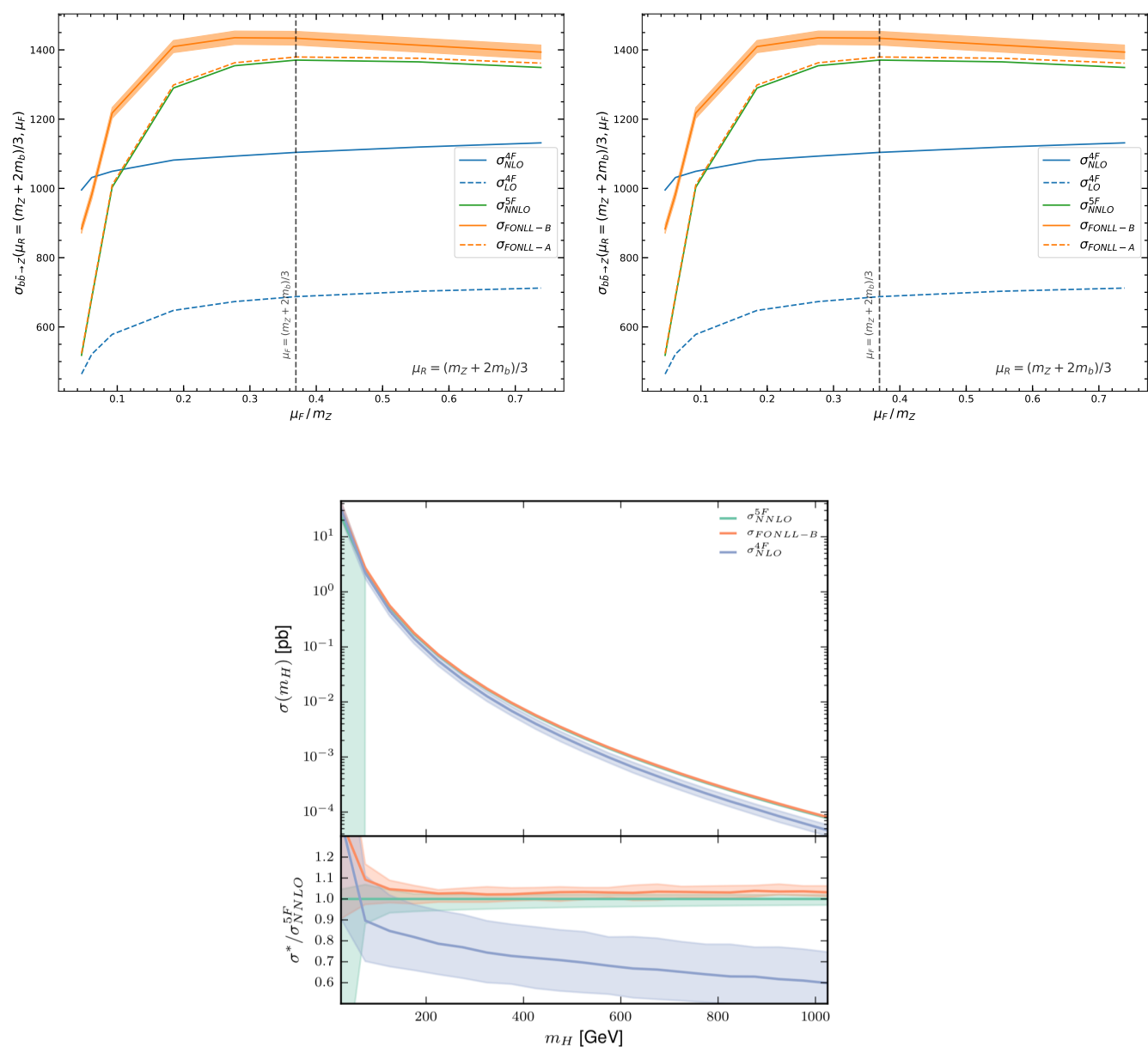

Figure 2: The FONLL matching scheme for the inclusive cross section of $b \bar{b} \rightarrow Z$ (Top row) as a function of $\mu_{R, F}$ and of $b \bar{b} \rightarrow H$ (Bottom row) as a function of $m_{H}$. Matched results are compared to the standard 4F and $5 \mathrm{~F}$ schemes.

accuracy, lies very close to the nominal five flavour scheme, thus concluding that mass effects play a little role, with the main difference between the two schemes, coming from the resummation of large logs.

The conclusion just shown seems to generally hold even in differential distributions for more exclusive observables [5]. Nevertheless, there are some regions of phase-space where the inclusion of fixed-order mass effects is more important than resummation. However, performing an analytical matching in these more exclusive cases is typically just not doable. For this reasons, we propose to extend a old idea, used mostly in DIS processes, to hadron-hadron collisions at NLO accuracy: retaining mass effects in the initial state for heavy quarks. We name this scheme, the five flavour massive scheme, or 5MS in short [9].

The main idea of this scheme, is to allow $b$ (or any other heavy) quarks in the initial state as in the $5 \mathrm{~F}$ scheme, yet retaining full mass dependence at the matrix element and phase-space level. This is done at NLO by extending Catani-Seymour [12, 13, 14] subtraction in the SHERPA Monte 
Carlo event generator, to allow for massive initial state $[9,15]$.

To see how the inclusion of such mass effects affects differential predictions, we studied for $b \bar{b} \rightarrow H$ at NLO fixed order accuracy and $b \bar{b} \rightarrow Z$ in a MEPs@NLO [16] simulation. Results are reported in Figs. 3 and 4 respectively.

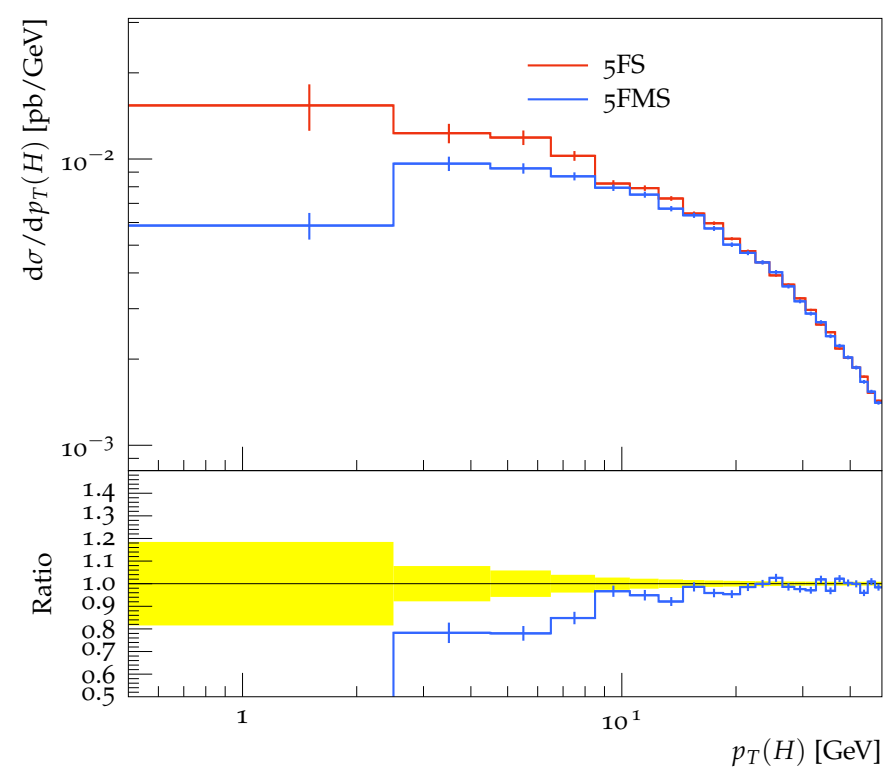

Figure 3: Comparison of the 5F and the 5FM scheme in $b \bar{b} \rightarrow H$ at NLO fixed order accuracy.

In Fig. 3 it can be seen how mass effects have an impact, and also quite a large one, only in the very low $p_{T}$ region. Once $p_{T}$ becomes of the order of about twice the bottom mass, these effects become negligible and the massless and massive schemes converge to each other, as expected.

Moving to Fig. 4. Here we generate a merged [18] sample of $Z$ plus 0,1,2 and 3 jets respectively. Further we look at events with at least one $b$ jet. This means that, on top of the bottom initiated contributions, we also have the light quark ones. The latter are typically much larger than the former, as their parton distribution function is much larger. In addition, because of experimental statistics, very low $p_{T}$ bins are integrated into larger bins than the ones shown for the fixed order case. For this reasons in this process we do not see any significant effect coming from the inclusion of mass effects.

Further details and studies on this proposed scheme can be found in [9]

\section{References}

[1] Fabio Maltoni, Giovanni Ridolfi, and Maria Ubiali. b-initiated processes at the LHC: a reappraisal. JHEP, 07:022, 2012. [Erratum: JHEP04,095(2013)].

[2] M. Wiesemann, R. Frederix, S. Frixione, V. Hirschi, F. Maltoni, and P. Torrielli. Higgs production in association with bottom quarks. JHEP, 02:132, 2015.

[3] Stefano Forte, Davide Napoletano, and Maria Ubiali. Higgs production in bottom-quark fusion in a matched scheme. Phys. Lett., B751:331-337, 2015. 

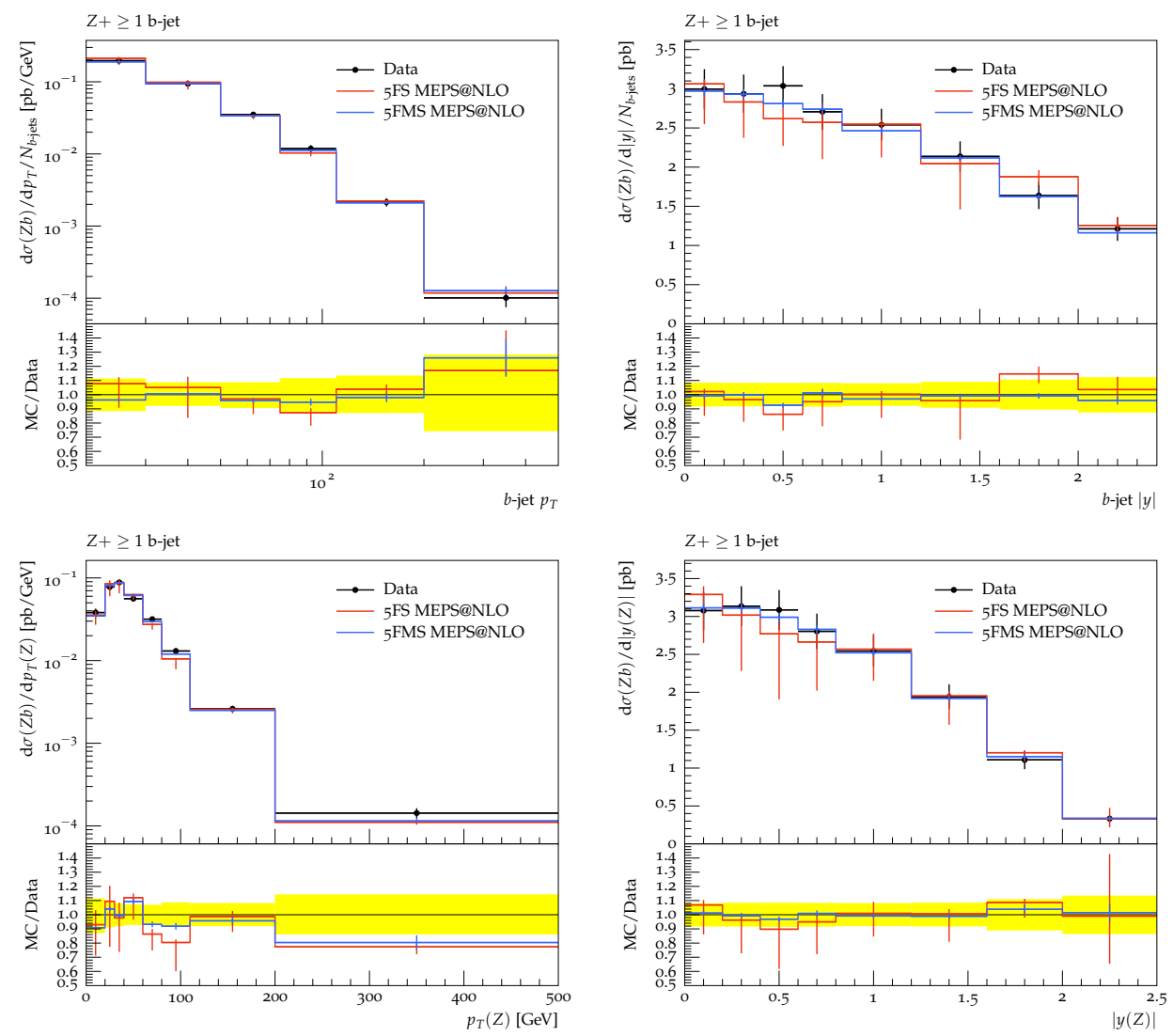

Figure 4: We show prediction obtained in the 5FS, massless, at MEPs@NLO accuracy, with up to 2 jets at NLO plus up to three jets at leading order. The 5FMS prediction on the other hand includes only the 0 jet contribution at NLO, while the 1,2 and 3 jets contributions are merged with LO accuracy, data from [17]

[4] Marco Bonvini, Andrew S. Papanastasiou, and Frank J. Tackmann. Resummation and matching of b-quark mass effects in $b \bar{b} H$ production. JHEP, 11:196, 2015.

[5] Frank Krauss, Davide Napoletano, and Steffen Schumann. Simulating $b$-associated production of $Z$ and Higgs bosons with the SHERPA event generator. Phys. Rev., D95(3):036012, 2017.

[6] Marco Bonvini, Andrew S. Papanastasiou, and Frank J. Tackmann. Matched predictions for the $b \bar{b} H$ cross section at the $13 \mathrm{TeV}$ LHC. JHEP, 10:053, 2016.

[7] Matthew Lim, Fabio Maltoni, Giovanni Ridolfi, and Maria Ubiali. Anatomy of double heavy-quark initiated processes. JHEP, 09:132, 2016.

[8] Stefano Forte, Davide Napoletano, and Maria Ubiali. Higgs production in bottom-quark fusion: matching beyond leading order. Phys. Lett., B763:190-196, 2016.

[9] Frank Krauss and Davide Napoletano. Towards a fully massive five-flavour scheme. 2017.

[10] Emanuele Bagnaschi, Fabio Maltoni, Alessandro Vicini, and Marco Zaro. Lepton-pair production in association with a $b \bar{b}$ pair and the determination of the $W$ boson mass. 2018.

[11] Stefano Forte, Davide Napoletano, and Maria Ubiali. $Z$ boson production in bottom-quark fusion: a study of $b$-mass effects beyond leading order. 2018. 
[12] S. Catani and M. H. Seymour. A general algorithm for calculating jet cross sections in NLO QCD. Nucl. Phys., B485:291-419, 1997.

[13] Stefano Catani, Stefan Dittmaier, Michael H. Seymour, and Zoltan Trocsanyi. The dipole formalism for next-to-leading order QCD calculations with massive partons. Nucl. Phys., B627:189-265, 2002.

[14] Stefan Dittmaier. A general approach to photon radiation off fermions. Nucl. Phys., B565:69-122, 2000.

[15] T. Gleisberg, Stefan. Hoeche, F. Krauss, M. Schonherr, S. Schumann, F. Siegert, and J. Winter. Event generation with SHERPA 1.1. JHEP, 02:007, 2009.

[16] Stefan Höche, Frank Krauss, Marek Schönherr, and Frank Siegert. NLO matrix elements and truncated showers. JHEP, 08:123, 2011.

[17] Georges Aad et al. Measurement of differential production cross-sections for a $Z$ boson in association with $b$-jets in $7 \mathrm{TeV}$ proton-proton collisions with the ATLAS detector. JHEP, 10:141, 2014.

[18] Stefano Catani, Frank Krauss, Ralf Kuhn, and Brian R. Webber. QCD matrix elements + parton showers. JHEP, 11:063, 2001. 